\title{
Linx
}

Revue des linguistes de l'université Paris X Nanterre

38 | 1998

L'acquisition de la temporalité en situation bilingue

\section{La langue faible (l'anglais) chez un enfant bilingue anglais-français}

Harriet Jisa

\section{(2) OpenEdition}

\section{Journals}

Édition électronique

URL : http://journals.openedition.org/linx/869

DOI : $10.4000 /$ linx. 869

ISSN : 2118-9692

\section{Éditeur}

Presses universitaires de Paris Nanterre

\section{Édition imprimée}

Date de publication : 1 décembre 1998

Pagination : 81-99

ISSN : 0246-8743

\section{Référence électronique}

Harriet Jisa, « La langue faible (l'anglais) chez un enfant bilingue anglais-français », Linx [En ligne],

38 | 1998, mis en ligne le 27 juin 2012, consulté le 30 avril 2019. URL : http://journals.openedition.org/ linx/869; DOI : 10.4000/linx.869

Ce document a été généré automatiquement le 30 avril 2019.

Département de Sciences du langage, Université Paris Ouest 


\title{
La langue faible (l'anglais) chez un enfant bilingue anglais-français
}

\author{
Harriet Jisa
}

Dans les études sur le bilinguisme précoce, on distingue entre «bilinguisme simultané » et « bilinguisme consécutif ». Certains chercheurs (cf. McLaughlin 1984, 1985) considèrent comme bilingues simultanés les enfants qui ont été en contact avec les deux langues avant l'âge de trois ans. D'autres encore (cf. De Houwer 1990, Padilla \& Lindholm 1984 , Meisel 1989) sont plus exigeants dans leur définition: ils considèrent comme bilingues simultanés uniquement les enfants qui ont été en contact avec les deux langues depuis la naissance, «bilinguisme comme première langue» («Bilingual First Language Acquisition » (Meisel 1989)). L'enfant étudié ici a été en contact quotidien avec les deux langues depuis sa naissance. Sa compréhension de l'anglais n'a jamais posé de problèmes mais sa production était, comme on l'a dit, très réduite.

On sait qu'à l'âge de trois ans un enfant est considérablement avancé dans l'acquisition de sa langue. Même pendant la première année de la vie, les nourrissons montrent certains comportements verbaux dans le babillage que l'on peut considérer comme spécifiques de la langue de l'entourage (de Boysson-Bardies, Sagart \& Durand 1984, Mehler, Lamertz, Jusczyk \& Amiel-Tison 1986). On ne peut donc pas écarter l'hypothèse que les enfants bilingues qui ont été exposés quotidiennement aux deux langues dès la naissance, et qui ont produit dans les deux langues, et ceux qui, dans un premier temps, n'ont produit que dans une seule des deux langues, suivent une trajectoire de développement différente.

Le travail présenté ici concerne le développement de l'auxiliaire $\mathrm{BE}$ en anglais pendant une période de deux mois lors d'un séjour en pays anglophone. Pendant cette période, la langue faible, l'anglais, s'est développée très rapidement. Nous verrons que les erreurs au cours de l'acquisition sont très différentes chez cette fillette si on les compare aux erreurs citées pour les enfants monolingues anglophones. Dans cet article nous allons examiner trois explications possibles pour cette différence. Deux formes seront étudiées en détail, AUX V-ing (I'm eating it) et AUX Vnon-fléchi ( ${ }^{*}$ 'm open the door). La deuxième forme, rarement observée chez les enfants anglophones monolingues, est très fréquente dans la 
production de la fillette étudiée ici. La première source d'erreur envisagée est que la forme V-ing est une forme figée, c'est-à-dire que certains verbes n'émergent que sous la forme avec -ing. La deuxième explication possible est que la sémantique des verbes est déterminante dans le choix entre AUX V-ing et AUX Vnon-fléchi. Une troisième source d'erreur peut se trouver dans un contraste temporel, c'est-à-dire que le choix de la fillette entre AUX V-ing et AUX Vnon-fléchi est déterminé par une distinction temporelle.

Nous allons d'abord tenter de définir la notion de «langue faible». Nous verrons que pour mieux saisir la définition du type de bilingue, il est important de prendre en compte des facteurs autres que l'âge auquel l'enfant est exposé aux deux langues. Puis nous décrirons brièvement le pattern d'erreurs observé chez Odessa. Enfin, nous présenterons les trois hypothèses pour en évaluer l'adéquation explicative. En conclusion, nous expliquerons comment la précocité de l'auxiliaire préverbal en anglais peut constituer un type de transfert de la langue forte, le français, à la langue faible, l'anglais.

\section{La langue faible}

5 Schlyter (1993: 69) propose un certain nombre de facteurs à prendre en compte pour l'évaluation précise de la compétence productive dans la langue faible :

$$
\text { une faible Longueur Moyenne des Énoncés (LMÉ) }
$$

l'absence de la maîtrise de certains traits développementaux constituant des paliers, comme les modaux, les subordonnées, le marquage de la référence au passé, la "syntaxisation " (Givon 1984)

la faible disposition à parler la langue (fluency), par exemple, combien de phrases autres que oui ou non sont produites dans la langue?

les mélanges, des lexèmes ou des énoncés entiers provenant de l'autre langue

une pauvreté du vocabulaire, par exemple, combien d'unités lexicales différentes sont utilisées?

le choix de la langue dans des contextes bilingues.

6 Avant son séjour en pays anglophone, la production anglaise d'Odessa remplissait tous les critères d'une langue faible. Le calcul de la longueur moyenne des énoncés n'était même pas possible dans la mesure où la quantité d'énoncés était trop faible. Le nombre total des énoncés en anglais autre que yes ou no dans les transcriptions des enregistrements couvrant les six mois précédant son séjour va de 2 à 22 énoncés, soit de $0.5 \%$ à $8 \%$ de la production totale. Les mélanges (qui varient entre $1 \%$ à $7 \%$ suivant la transcription) dans ces mêmes transcriptions consistent en l'emploi de quelques noms anglais dans des phrases françaises. De plus, le vocabulaire productif anglais était très restreint : outre les formes figées mentionnées plus haut, le vocabulaire anglais se limitait à quelques mots pour les vêtements (shoe, sock), les couleurs (pink, orange, yellow, blue) et les formes géométriques (square, triangle). Le choix du français dans des contextes bilingues (qui sont d'ailleurs les seuls contextes auxquels la fillette participe) est très clair: le français représente $79 \%$ à $90 \%$ du total des énoncés (autre que oui/yes, non/no) produits pendant les six mois précédant son séjour. 


\section{Le sujet : Odessa}

Depuis la naissance d'Odessa, sa mère lui a toujours parlé en anglais et son père en français. Dès l'âge de 3 mois elle a passé cinq à six heures par jour du lundi au vendredi dans une crèche monolingue francophone. Jusqu'à l'âge de $2 ; 9$, Odessa n'a été exposée qu'à l'anglais de sa mère. A $2 ; 9$, Odessa a commencé à fréquenter la maternelle dans une école bilingue anglais-français où elle passait une demi-journée en anglais et une demijournée en français. Les instituteurs/trices de la section anglaise, tous d'origine britannique, s'adressaient aux enfants en anglais mais n'exigeaient pas que les enfants s'expriment toujours en anglais.

Depuis l'âge de $2 ; 7$ Odessa a été enregistrée sur magnétophone et caméscope pendant deux heures à peu près toutes les deux semaines. Les participants à ces interactions sont Odessa, sa sœur cadette ( 15 mois plus jeune qu'Odessa), la mère et/ou le père. Les transcriptions analysées ici proviennent du premier séjour en pays anglophone. La fillette a été enregistrée presque tous les jours pendant deux mois. Les participants à ces enregistrements sont Odessa, sa soeur cadette, la mère, et des anglophones monolingues membres de la famille ou amis de la mère. Le père n'a pas séjourné avec la famille pendant les deux mois.

9 Le Diagramme 1 retrace le changement dans la production linguistique d'Odessa pendant les deux mois. Le séjour de deux mois est découpé en tranches de dix jours. Ainsi, « Temps $1 »$ fait référence aux dix premiers jours, « Temps 2 » aux dix jours suivants, etc.

Diagramme 1. Odessa : Courbes de développement

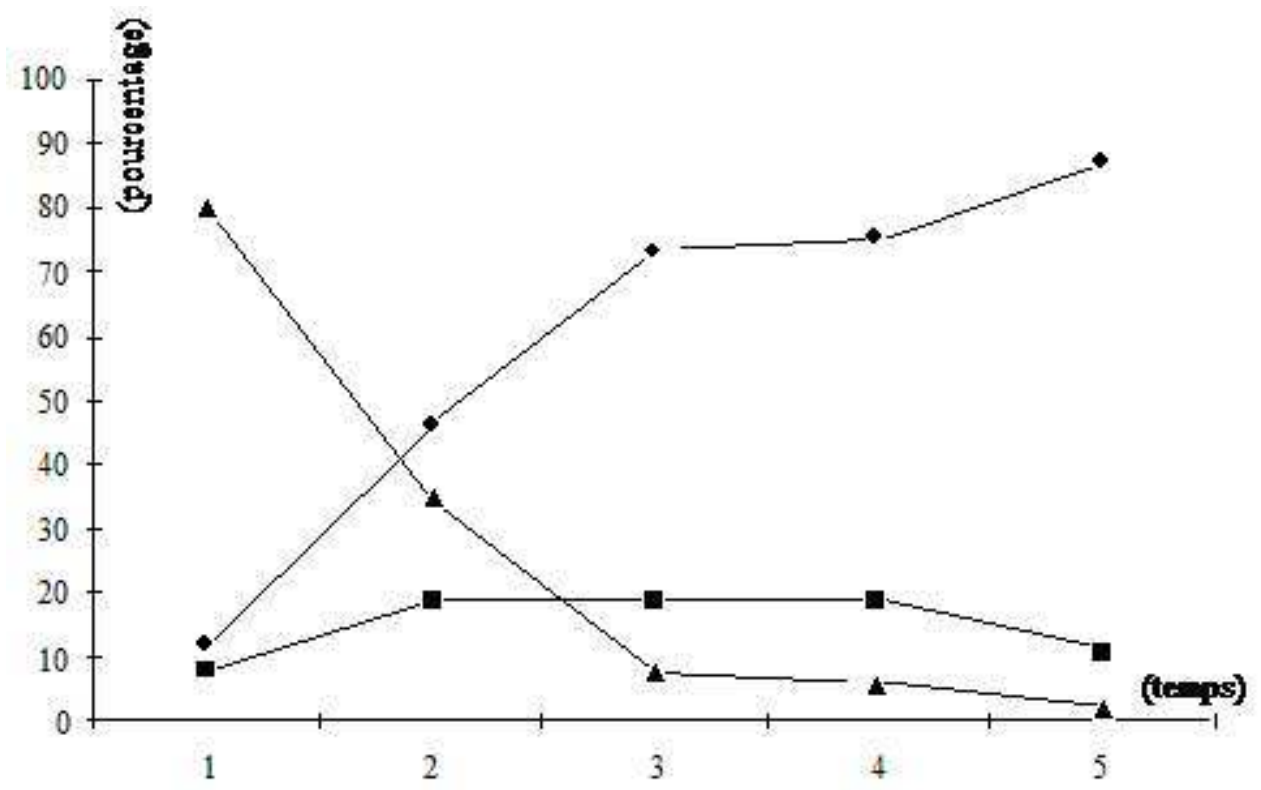

s français

I anglais

n mixte

Les transcriptions utilisées pour cette étude sont présentées dans le Tableau 1. «Date » fait référence à la date de l'enregistrement. Toutes les cassettes utilisées sont de 90 minutes. Certains enregistrements couvrent plus d'un jour. La deuxième colonne donne le 
nombre total de phrases anglaises, sans tenir compte des énoncés consistant en yes/no/ yeah/ok seulement, ni les chansons enfantines, ou des routines comme le comptage d'objets. Les auto-répétitions directement adjacentes sont également exclues. La troisième colonne donne le pourcentage des phrases anglaises sur le total de phrases de la transcription (anglaises, françaises, mixtes). LME fait référence à la longueur moyenne d'énoncés pour les phrases anglaises, calculée d'après les indications de Brown (1973). Cependant, contrairement au principe d'utiliser 100 énoncés, toutes les phrases anglaises ont été utilisées pour calculer la LME. La cinquième colonne donne l'écart entre la phrase la plus courte et la phrase la plus longue. « Moyenne des 5 phrases les plus longues » est la longueur moyenne d'énoncé (en morphèmes) calculée sur les cinq phrases anglaises les plus longues de la transcription. La dernière colonne indique comment les données ont été divisées en périodes. Les périodes ont été établies de façon à avoir à peu près le même nombre de phrases dans chaque période (Période II - 620 phrases, Période III - 664 phrases, Période IV - 658 phrases). Les deux premières transcriptions ont été regroupées dans la Période I. La LME étant inférieure à 2, la morphologie grammaticale est quasiinexistante.

Tableau 1. Odessa : la production anglaise

\begin{tabular}{|l|l|l|l|l|l|l|}
\hline Date & $\begin{array}{l}\text { Nombre } \\
\text { d'énoncés anglais }\end{array}$ & $\begin{array}{l}\text { \%d'énoncés } \\
\text { anglais / } \\
\text { total d'énoncés }\end{array}$ & LME & Etendue & $\begin{array}{l}\text { Moyenne des 5 phrases } \\
\text { les plus longues }\end{array}$ & Période \\
\hline $14-15.7$ & 72 & $19 \%$ & 1,3 & $1-3$ & 3 & I \\
\hline 16.7 & 29 & $6 \%$ & $(1,4)$ & $1-5$ & 3,2 & \\
\hline $25-28.7$ & 154 & $45 \%$ & 2,09 & $1-7$ & 5,4 & \\
\hline $1-2.8$ & 94 & $39 \%$ & 2,19 & $1-7$ & 5,4 & II \\
\hline $8-9.8$ & 117 & $72 \%$ & 2,8 & $1-9$ & 6,8 & IV \\
\hline $9.8 . I$ & 177 & $59,5 \%$ & 2,56 & $1-9$ & 6,6 & \\
\hline $9.8 . I I$ & 178 & $59 \%$ & 3,35 & $1-8$ & 7,4 & \\
\hline 13.8 & 240 & $78,5 \%$ & 3,32 & $1-11$ & 8,2 & \\
\hline 15.8 & 210 & $69 \%$ & 3,33 & $1-9$ & 8,2 & \\
\hline $15-19.8$ & 214 & $74,5 \%$ & 3,66 & $1-9$ & 8,2 & \\
\hline $22-23.8$ & 460 & $81 \%$ & 3,45 & $1-9$ & 9,2 & \\
\hline $23-25.8$ & 198 & 3,31 & 1.9 & 8,6 & \\
\hline
\end{tabular}

11 Bien que l'utilisation de la LME pose un certain nombre de problèmes, elle reste un des meilleurs indices du développement morpho-syntaxique (au moins pour les valeurs de 
LME inférieures à 4.50) (Meisel 1989, Rondal 1983). Contrairement à l'âge, la LME est corrélée positivement avec d'autres indices du développement morpho-syntaxique (de Villiers \& de Villiers 1978, Moerk 1977, Rondal 1983). L'un des avantages de la LME est qu'elle permet la comparaison entre enfants. En début de séjour, la LME d'Odessa est largement inférieure à celle des sujets monolingues de son âge, correspondant aux LMEs des enfants monolingues de 16 à 23 mois (de Villiers \& de Villiers 1973, Retherford, Schwartz \& Chapman 1981, Seitz \& Stewart 1975). A la fin de son séjour, la LME d'Odessa correspond à celle d'enfants monolingues de 30 à 40 mois (Cunningham, Reuler, Blackwell \& Deck 1981, Chapman 1981). L'augmentation extrêmement rapide de la LME en anglais chez Odessa montre que, lorsqu'un enfant bilingue se trouve placé dans un contexte où la langue faible devient la langue dominante de l'environnement, la compétence passive accumulée avant la période de contact intense se manifeste assez facilement par une certaine compétence productive.

Ramer (1976) compare le développement «lent» ou «rapide» chez sept enfants monolingues anglophones. Le temps nécessaire au passage de productions consistant en des combinaisons de deux mots à des productions consistant en au moins $20 \%$ de structures Sujet-Verbe-Complément était de six à neuf mois pour les enfants « lents » et de six semaines à quatre mois pour les enfants « rapides ». Plus de $20 \%$ des productions anglaises d'Odessa à la Période IV, six semaines après le début du séjour, sont des constructions Sujet-Verbe-Complément.

\section{BE : copule et auxiliaire}

13 Contrairement à ce qui se passe chez les enfants monolingues, l'utilisation de l'auxiliaire $\mathrm{BE}$ et de la copule $\mathrm{BE}$ dans les productions d'Odessa est très précoce, très fréquente et sujette à un certain nombre de surgénéralisations qui seront présentées ci-dessous. Selon Brown (1973) et De Villiers \& de Villiers (1973), l'auxiliaire est acquis après le passé régulier et irrégulier, après le présent et après le progressif. Cependant, chez Odessa l'auxiliaire est utilisé comme marqueur de l'aspect alors que le présent simple, par exemple, n'est pas productif de tout. Et nous verrons que l'auxiliaire BE est utilisé à la place de l'auxiliaire HAVE (du parfait), normalement considéré comme étant acquis tardivement (Cromer 1968, de Villiers \& de Villiers 1985).

Afin d'isoler les erreurs, nous avons fait appel à la notion de « contexte obligatoire » bien qu'elle ne soit pas toujours facilement applicable aux données spontanées. Pour l'étude présentée ici trois critères linguistiques et non-linguistiques ont été pris en compte afin de déterminer les contextes obligatoires (Brown 1973, Bloom, Lifter \& Hafitz 1980, Richards 1990). D'abord, le contexte linguistique de l'énoncé de l'enfant peut rendre obligatoire l'utilisation d'une forme fléchie. Dans (1) la présence de l'auxiliaire ' $m$ rend obligatoire la présence de -ing sur le verbe.

(1) I'm play horsey (14.7.86) (= I’m playing horsey)

Ensuite, le contexte conversational exige l'utilisation d'une forme fléchie. Dans (2), la question posée par l'adulte impose l'utilisation du présent progressif chez l'enfant. 
\begin{tabular}{|l|l|}
\hline (2) & A: What are you doing now Odessa? \\
\hline & O : I'm cooking chicken $(9.8 .86)$ \\
\hline
\end{tabular}

Enfin le contexte non-linguistique peut exiger l'utilisation d'une forme fléchie. Dans (3), étant donné le caractère achevé de l'action, le morphème du passé est obligatoire, mais n'est pas utilisé.

(3) O: I'm all finish! (2528.7.86) (après avoir terminé une glace) (=I'm all finished)

17 Deux catégories d'erreurs ont été déterminées. Les " erreurs d'omission » sont les cas où l'enfant n'a pas utilisé le morphème obligatoire (4a et $4 \mathrm{~b})$. Les " erreurs de commission" sont les cas où l'enfant a employé un morphème qui n'était pas nécessaire (4c) et où l'enfant a utilisé un morphème de façon erronée, comme dans (4d) où l'auxiliaire est utilisé sans inversion.

\begin{tabular}{|l|l|l|}
\hline (4) & a. & I'm all finish (25-28.7.86) (forme adulte: I'm all finished) \\
\hline \hline & b. & why the doggie there? (8-9.8.86) (forme adulte: why is the doggie there?) \\
\hline & c. & 'coz he's eats (8-9.8.86) (forme adulte: 'coz he eats or 'coz he's eating) \\
\hline & d. & when he's coming? (8-9.8.86) (forme adulte: when is he coming?) \\
\hline
\end{tabular}

Pour le travail présenté ici, seules les phrases affirmatives seront prises en considération.

\section{L'auxiliaire BE : présent progressif}

Le Tableau 5 indique les utilisations du présent progressif dans les phrases affirmatives. Dès la Période II, deux formes de l'auxiliaire ('s et ' $m$, ce dernieravec une variante phonétique $\mathrm{y})$ sont présentes. A la Période II is (l'auxiliaire non contracté) est utilisé une fois. A la Période IV, la forme contractée 're (2ème personne) et la forme non-contractée is sont utilisées chacune une fois. Dans la dernière colonne du Tableau 5 est indiqué le pourcentage d'insertion de l'auxiliaire dans les constructions avec -ing. Si on utilise le critère de $90 \%$ d'utilisations appropriées dans des contextes obligatoires (Brown 1973), l'auxiliaire est maîtrisé dès la Période II. Le morphème -ing sur le verbe principal, lui, ne l'est pas.

Tableau 5. Présent progressif : AUX V+ing vs. AUX Vnon-fléchi

\begin{tabular}{|l|l|l|l|l|l|}
\hline Période & Appropriée & Erreurs* & Total & $\begin{array}{l}\text { \% correct } \\
\text { AUX + V-ing }\end{array}$ & \%AUX \\
\hline
\end{tabular}




\begin{tabular}{|l|l|l|l|l|l|l|}
\hline & & I & II & & & \\
\hline \hline I & 0 & 1 & & 1 & & \\
\hline II & 12 & 12 & 1 & 24 & $50 \%$ & $95 \%$ \\
\hline III & 17 & 23 & & 40 & $42,5 \%$ & $100 \%$ \\
\hline IV & 11 & 5 & & 16 & $69 \%$ & $100 \%$ \\
\hline
\end{tabular}

*Type d'Erreur:

I S aux Vracine, ex. I'm eat, he's eat

II S V-ing, ex. I eating

20 Le morphème du progressif -ing est toujours mentionné comme étant parmi les premiers morphèmes acquis chez les enfants monolingues (Brown 1973, Fletcher 1985, Richards 1990). Par contre l'auxiliaire $\mathrm{BE}$ ( ( être ») qui accompagne le morphème -ing et porte le temps est normalement maîtrisé plus tard. L'erreur typique des anglophones monolingues consiste en l'utilisation de -ing sans l'auxiliaire (Erreur de type II). Les productions d'Odessa montrent un phénomène inverse. L'auxiliaire contracté est présent massivement dès la Période II. Par contre le -ing fait défaut même à la Période IV et constitue l'erreur (Erreur de type I) la plus représentée dans les productions d'Odessa. Ces résultats diffèrent considérablement de ceux obtenus sur les sept enfants étudiés par Richards (1990) où l'utilisation de l'auxiliaire dans des contextes obligatoires ne dépassait pas $45 \%$, bien que le -ing ne soit pas mentionné comme posant problème.

21 Un certain nombre d'hypothèses ont été envisagées afin d'expliquer pourquoi l'enfant n'utilise pas systématiquement le morphème -ing. La première, «l'hypothèse de la forme figée ", propose que l'enfant n'a qu'une forme par verbe, c'est-à-dire que le morphème ing est analysé comme faisant partie de la racine du verbe. La deuxième, «l'hypothèse sémantique ", postule que la présence ou l'absence du morphème -ing est déterminée par la sémantique du verbe. L'« hypothèse du contraste temporel » propose que l'enfant fait une distinction temporelle entre des événements encodés par les structures AUX Vnonfléchi (Erreur de type 1) et par la structure appropriée, AUX V-ing.

\section{L'hypothèse de la forme figée}

La première hypothèse envisagée propose que les formes verbales sont des formes figées, c'est-à-dire que l'enfant analyse la forme V-ing comme la forme de la racine verbale. Un des arguments nécessaire pour défendre cette hypothèse est que l'enfant n'utilise qu'une forme (V-ing) pour une racine verbale donnée. Selon l'hypothèse de la masse critique (Marchman \& Bates 1984) cette situation serait possible pour de très jeunes anglophones qui n'ont que quelques verbes et qui ne sont qu'au début de leur analyse des unités en racine + affixe.

23 Afin d'évaluer cette hypothèse, toutes les premières utilisations de verbes produits avec le morphème -ing ont été isolées, puis codées selon quatre critères : 1) le verbe n'a JAMAIS été utilisé sous une autre forme durant le séjour ;2) le verbe a été utilisé sous une autre forme à une PÉRIODE ULTÉRIEURE ; 3) le verbe a été utilisé sous une autre forme dans la 
MÊME PÉRIODE ; et 4) le verbe a été utilisé sous une autre forme à une PÉRIODE PRÉCÉDENTE. Les résultats de ce codage se trouvent dans le Tableau 6.

Tableau 6. Utilisation de verbes avec -ing et sous d'autres formes

\begin{tabular}{|c|c|c|c|c|}
\hline & jamais & Période ultérieure & même Période & Période précédente \\
\hline \multirow[t]{4}{*}{ Période II } & cooking & coming & looking & crying \\
\hline & screaming & & scratching & working \\
\hline & sleeping & & taking & \\
\hline & swimming & & & \\
\hline \multirow[t]{3}{*}{ Période III } & chasing & & changing & falling \\
\hline & & & eating & reading \\
\hline & & & going & \\
\hline \multirow[t]{2}{*}{ Période IV } & fighting & & trying & sitting \\
\hline & smoking & & & turning \\
\hline
\end{tabular}

Les résultats donnés dans le Tableau 6 ne semblent pas indiquer qu'Odessa attribue aux formes Ving un statut de forme figée. Pendant la Période I, aucun verbe n'est employé sous la forme Ving. A la Période II, cinq verbes ont émergé sous la forme Ving. Un de ces verbes (coming) est employé sous une autre forme à une période ultérieure. Un nombre égal d'autres verbes est utilisé sous une autre forme soit dans la même période, soit à une période précédente. Il semble donc que dès la Période II -ing doive être considéré comme un morphème productif et pas comme faisant partie d'une forme figée.

\section{L'hypothèse sémantico-aspectuelle}

L'hypothèse sémantico-aspectuelle prédit une différence dans la distribution des formes selon la sémantique des verbes. Si l'enfant établit une distribution complémentaire entre les structures AUX V-ing et AUX Vnon-fléchi selon le lexème verbal utilisé, il serait possible d'envisager que la sémantique des unités lexicales ou aspect lexical joue un rôle dans l'élaboration du système. Afin d'évaluer cette hypothèse, nous avons examiné les verbes employés, soit sous les deux formes, soit uniquement sous la forme AUX V-ing, ou dans la forme AUX Vnon-fléchi. Le Tableau 7 donne, pour chaque période, les résultats de cette analyse. 
Tableau 7. Distribution des verbes utilisés avec les structures AUXV-ing et AUX V

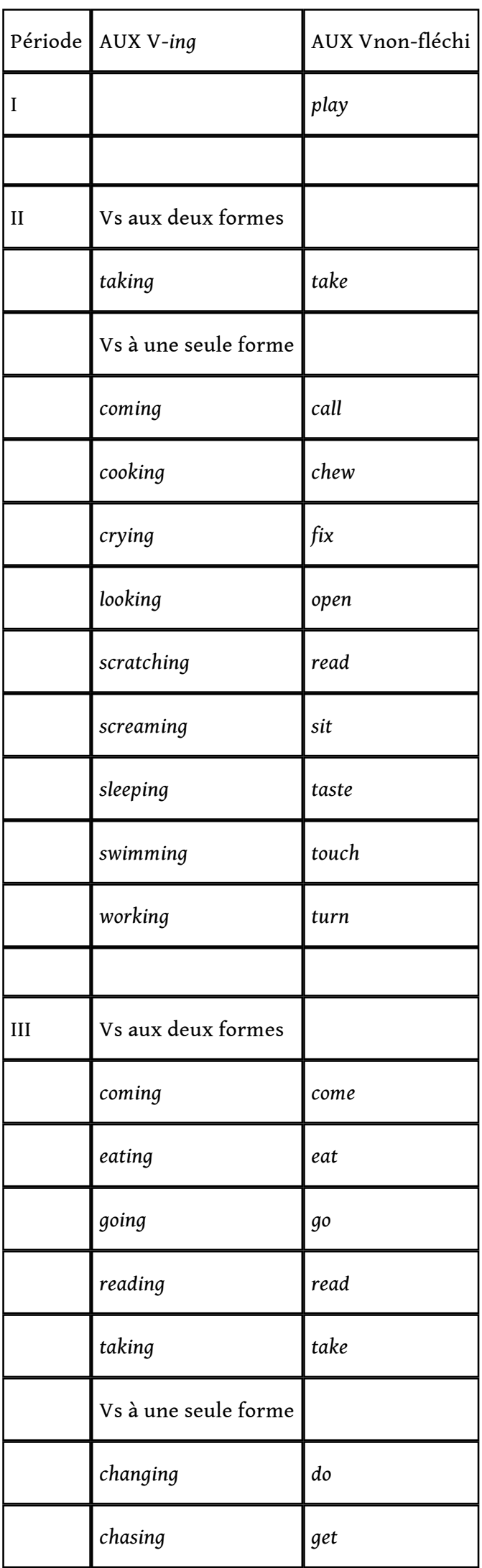




\begin{tabular}{|c|c|c|}
\hline & going & put \\
\hline & falling & taste \\
\hline & working & throw \\
\hline & watch & \\
\hline \multirow[t]{10}{*}{ IV } & Vs aux deux formes & \\
\hline & trying & try \\
\hline & Vs à une seule forme & \\
\hline & coming & close \\
\hline & crying & go \\
\hline & eating & open \\
\hline & fighting & play \\
\hline & sitting & \\
\hline & smoking & \\
\hline & turning & \\
\hline
\end{tabular}

Le Tableau 7 montre qu'il y a variation pour un même verbe (take/taking) dès la Période II. A la Période III, plusieurs verbes sont employés sous les deux formes. Il ne semble pas que la présence ou absence de -ing soit déterminée par le verbe en tant qu'unité lexicale.

Cependant, les données montrent que les deux formes employées par Odessa diffèrent dans leur caractère " télique/atélique ", défini comme la " distinction entre un processus ou un événement conçus comme impliquant un seuil à franchir et un processus ou un événement conçus sans un seuil à franchir » (Creissels 1995 : 177). Cette distinction entre situation » télique » et situation « atélique » (cf. Comrie 1976:44-48, Vendler $1967: 102$ ) semble contribuer à l'explication de la distribution des formes AUX V et AUX V-ing. Cette distinction ne repose pas sur le seul choix du verbe (Comrie 1976: 45, Croft 1990: 77, Hopper 1979). Il est nécessaire de prendre en compte tout l'énoncé décrivant la situation. Ainsi il est possible d'utiliser un verbe indiquant un processus duratif pour décrire une situation atélique ( $5 \mathrm{a}$ et $\mathrm{b}$ ) ou une situation télique ( $6 \mathrm{a}$ et $b$ )

\begin{tabular}{|l|l|l|}
\hline$(5)$ & a. & Jean a chanté. \\
\hline & b. & Jean a bu du vin \\
\hline
\end{tabular}




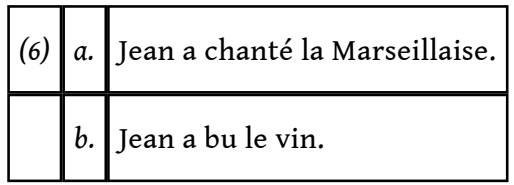
trouvent dans le Tableau 8. En ce qui concerne les verbes téliques (throw out, finish, fix, stop) 96 \% (47/49) se trouvent sous la forme AUX Vnonfléchi. La forme AUX V-ing semble attirer les verbes atéliques (working, crying, eating, cooking). $72 \%(50 / 69)$ des verbes atéliques prennent cette forme, contre $28 \%$ (19/69) sous la forme AUX Vnon-fléchi.

Tableau 8. Distribution des formes AUX V-ing et AUX Vnon-fléchi selon le caractère télique / atélique de la situation

\begin{tabular}{|l|l|l|l|}
\hline & & Atélique & Télique \\
\hline AUX V-ing & Période II & 15 & 1 \\
\hline & Période III & 21 & 0 \\
\hline & Période IV & 14 & 1 \\
\hline Total & & 50 & 2 \\
\hline & & & \\
\hline AUX Vnon-fléchi & Période II & 4 & 10 \\
\hline & Période III & 12 & 31 \\
\hline & Période IV & 3 & 6 \\
\hline Total & & 19 & 47 \\
\hline
\end{tabular}

Bien qu'il soit possible de voir dans ces résultats un effet de la sémantique lexicale sur les formes choisies, il reste cependant $28 \%$ des situations atéliques qui prennent la forme AUX Vnon-fléchi. Nous verrons dans la partie suivante qu'il est nécessaire de considérer, outre les caractères sémantiques et aspectuels des situations, des traits liés aux distinctions temporelles. 


\section{L'hypothèse du contraste temporel}

L'hypothèse du contraste temporel prédit une différence dans les relations temporelles auxquelles renvoient les deux formes en question. Les événements relatés par les structures AUX V-ing sont des actions en cours au moment de la parole. Des exemples typiques sont donnés en (7). Ces constructions progressives sont conformes à la langue cible qui exige l'utilisation de -ing sur le verbe principal.

\begin{tabular}{|l|l|l|}
\hline (7) & a. & I'm eating it (en réponse à sa mère qui demandait à Odessa de manger sa salade) \\
\hline & b. & $\begin{array}{l}\text { I'm playing now ( = I'm playing with it now) (en réponse à sa soeur qui voulait un jouet avec } \\
\text { lequel Odessa était en train de jouer) }\end{array}$ \\
\hline
\end{tabular}

Les événements relatés par les structures AUX Vnon-fléchi débutent au moment de la parole et «annoncent » en quelque sorte un événement à venir. Des exemples typiques sont donnés en (8).

\begin{tabular}{|l|l|l|}
\hline (8) & a. & I'm play with this (dit en prenant un jouet à sa soeur) \\
\hline & b. & I'y open the door (dit en s'approchant de la porte) \\
\hline & c. & I'y get down (dit en quittant sa chaise) \\
\hline
\end{tabular}

Dans la langue cible, (8a-c) seront normalement encodés avec le semi-auxiliaire « gonna » (< « going to ») et s'opposent aux structures AUX V-ing (8a-b), qui encodent une action en cours de réalisation. Conformément à la langue cible, Odessa utilise le verbe principal sans désinence dans les contextes appropriés au semi-auxiliaire gonna pour encoder le futur proche, bien que la réalisation observée chez Odessa soit homophonique avec l'auxiliaire BE. Il est à noter aussi que la variante nasale vélaire de l'auxiliaire contracté [y] se trouve uniquement dans les contextes du futur proche. Une autre preuve du fait que l'auxiliaire dans les structures AUX Vnon-fléchi (8) est le précurseur de gonna est que ce dernier commence à être produit pour le futur proche à la Période III ( 2 fois) et à la Période IV (4 fois).

Un deuxième contexte temporel où l'on constate la forme AUX Vnon-fléchi se trouve dans l'expression des événements au passé. On trouve deux structures dans les productions d'Odessa pour encoder le passé : le «simple past» et une structure que nous appelons «parfait» («present perfect») (Tableau 9). Le «simple past» ne semble toujours pas acquis à la Période IV. Des 18 mentions d'événements au passé, seules $9(50 \%)$ sont appropriées. Aucune erreur de surgénéralisation du morphème -ed à des verbes irréguliers (par exemple, I goed pour I went) n'est constatée. Cette erreur de surgénéralisation est souvent observée chez les enfants monolingues et constitue une preuve de l'acquisition du morphème -ed (de Villiers \& de Villiers 1985, Wells 1985). La seule erreur observée est l'utilisation d'un verbe non-fléchi (par exemple *she push herself 
( = she pushed herself), ${ }^{*}$ I fall down ( = I fell down)), erreur relevée aussi chez les enfants monolingues (Wells 1985).

La deuxième structure utilisée pour exprimer des événements au passé est l'auxiliaire $\mathrm{BE}$ avec un verbe non-fléchi ou un participe passé. Il n'est pas toujours possible d'évaluer dans les productions s'il s'agit du début de l'émergence de have comme auxiliaire (*she's finish, avant contraction 'she has finished', après contraction 'she's finished') ou de l'utilisation de la copule plus un complément adjectival (it's broken/I'm tired/mine is gone). Cependant l'utilisation de BE + Vnon-fléchi (Tableau 9, Erreur) ou BE + Vparticipe passé (Tableau 9, Approprié) semble une façon productive d'encoder le passé.

Nous allons tenter de défendre la thèse selon laquelle les structures AUX Vnon-fléchi sont en fait les précurseurs du parfait ("present perfect», have + participe passé). Deux problèmes sont posés dans l'interprétation de la structure AUX Vnon-fléchi. L'auxiliaire have est sujet lui aussi à la contraction. Dans la langue cible toutes les personnes (sauf la 3ème personne du singulier) ont la même forme, have, qui devient après contraction 've. La forme de la troisième personne, has, devient après contraction 's (he has fallen $\rightarrow$ he's fallen) et est donc un homonyme de BE (auxiliaire et copule). Pour cette raison, dans le Tableau 9 la première et la troisième personne sont séparées. Le deuxième problème concerne la forme du verbe principal. Pour les verbes réguliers, la forme du verbe est la même pour le «simple past» et le parfait, I painted («simple past») vs. I have painted (perfectif). Mais pour les verbes irréguliers ces deux formes sont distinctes, I fell, I have fallen, I did, I have done. Les structures BE + Vparticipe passé (par ex., mine is all gone) sont indiquées comme « Appropriées » dans le Tableau 9.

Tableau 9. L'utilisation de BE dans les formes AUX Vparticipe passé et AUX Vnon-fléchi pour exprimer des événements au passé

\begin{tabular}{|l|l|l|l|l|l|l|l|l|l|}
\hline & \multicolumn{3}{l}{ Approprié } & \multicolumn{4}{l}{ Erreur* } & Total \\
\hline Période & \multicolumn{2}{l}{ régulier } & \multicolumn{2}{l}{ irrégulier } & \multicolumn{2}{l|}{ régulier } & \multicolumn{2}{l|}{ irrégulier } & \\
\hline & $1^{\mathrm{e}} \mathrm{ps}$ & $3^{\mathrm{e}} \mathrm{ps}$ & $1^{\mathrm{e}} \mathrm{ps}$ & $3^{\mathrm{e}} \mathrm{ps}$ & $1^{\mathrm{e}} \mathrm{ps}$ & $3^{\mathrm{e}} \mathrm{ps}$ & $1^{\mathrm{e}} \mathrm{ps}$ & $3^{\mathrm{e}} \mathrm{ps}$ & \\
\hline I & 1 & & & & 1 & & & 1 & 3 \\
\hline II & 1 & & & & 3 & & 5 & & 9 \\
\hline III & 2 & & & 1 & 7 & 1 & & & 11 \\
\hline IV & & 2 & & & 2 & 2 & & & 6 \\
\hline
\end{tabular}

*Type d'Erreur:

S BE Vnon-fléchi, the bread is fall down, I'm fall down on my mouth

Un certain nombre d'arguments peuvent être envisagés en faveur d'une analyse de la structure BE + Vnon-fléchi comme précurseur du parfait («present perfect »), c'est-à-dire que la catégorie AUX est en place, mais qu'une forme déjà acquise - l'auxiliaire BE - est utilisée au lieu de HAVE. Un premier argument concerne l'utilisation de cette forme en réponse à des questions de l'adulte employant have, comme dans (9). 


\begin{tabular}{|l|l|l|}
\hline$(9)$ & & (avant de partir en voyage) \\
\hline \hline & A: & have you guys gone pipi? (« avez-vous fait pipi »?) \\
\hline & O: & I'm go pipi. («j'ai fait pipi ») \\
\hline
\end{tabular}

Le deuxième argument concerne le sens aspectuel à attribuer à ces formes. Lyons (1977) oppose le «simple past » au parfait (" present perfect»). Le «simple past» indique une action passée à un moment défini dans le passé. Le parfait (" present perfect ») indique une action dans le passé (achevé) qui reste pertinente pour la situation immédiate (résultatif). (10a) relate un événement passé (achevé) et fonctionne en fait comme demande adressée à sa tante de ramasser le pain, autrement dit de remédier au résultat de l'événement achevé. (10b) est en fait un appel au secours après une chute.

\begin{tabular}{|l|l|l|}
\hline$(10)$ & a. & the bread is fall down («le pain est tombé ») \\
\hline & b. & I'm fall down on my mouth («j'ai tombé sur ma bouche ») \\
\hline
\end{tabular}

Bien qu'il n'y ait pas de preuve qu'Odessa maitrise le participe passé, il semble qu'elle utilise $\mathrm{BE}$ dans des contextes où l'auxiliaire have est approprié dans la langue cible.

Fletcher (1985 : 123-127) décrit longuement une erreur très fréquente chez son sujet âgé de $3 ; 00$, Sophie. Cette erreur consiste en l'utilisation du morphème -ed (« simple past ») et du morphème -en (participe passé), sans auxiliaire pour encoder un événement du passé, par exemple I eaten pour «I ate ». Il évoque une hypothèse sémantique: -en serait restreint aux événements du passé récent (défini comme précédant le moment de la parole de cinq minutes ou moins) et -ed serait utilisé pour un intervalle de temps plus éloigné. D'après l'examen des données, il rejette cette hypothèse : les deux morphèmes sont utilisés pour relater des événements récents.

Nous avons envisagé cette hypothèse temporelle comme explication pour la variation chez Odessa entre les formes V-ed (I painted), *Vnon-fléchi (*she push herself) et *AUX Vnon-fléchi ( ${ }^{*} I ' m$ fall down) pour encoder le passé. La majorité des références au passé d'Odessa et de Sophie sont des références au passé récent. Toutefois, lorsqu'Odessa fait référence au passé éloigné (allant de plus d'une heure avant le moment de la parole à plus de 24 heures avant), elle utilise la forme V-ed (I painted) 9 fois sur 10. Toutes les formes BE Vfléchi (mine is all gone, cf. les utilisations appropriées sur le Tableau 9) et les formes $\mathrm{SBE}$ Vnon-fléchi (*I'm finish, cf. les utilisations erronées sur le Tableau 9) relatent les événements du passé récent. Nous proposons ici que les formes BE Vnon-fléchi utilisées pour exprimer le passé sont des précurseurs de la forme HAVE Vparticipe passé (" present perfect »), tandis que les formes S BE Vfléchi sont des formes de la copule + un complément adjectival. Autrement dit, la même forme ancienne - BE - remplit deux fonctions, dont une sera ensuite remplacée par une nouvelle forme : HAVE. 


\section{Discussion}

43

Vnonfléchi. Nous avons écarté la première, l'hypothèse de la forme figée : la distribution des verbes portant le morphème -ing et des verbes sans le morphème n'est pas complémentaire. Le même verbe apparaît soit avec, soit sans -ing. Notre examen de l'hypothèse portant sur la distinction aspectuelle télique /atélique revèle que la forme AUX V-ing est attirée par les situations atéliques. L'examen de l'hypothèse du contraste temporel nous a montré que les formes AUX Vnonfléchi sont employées pour encoder 1) les actions du futur immédiat et 2) des événements passés dont le résultat reste pertinent pour l'immédiat. Nous avons proposé que la première utilisation évolue vers l'utilisation de gonna, et que la deuxième évoluera vers l'utilisation de have. témoignent d'une différence considérable entre le développement de l'anglais chez cette fillette et les enfants anglophones monolingues. Odessa n'a pas attribué au morphème ing la saillance que lui attribuent les enfants monolingues. Par contre, elle a attribué une certaine saillance à l'auxiliaire. Dans ce qui suit, nous allons proposer que le pattern de développement d'Odessa peut être attribué au français, sa langue forte. ${ }^{1}$

En français, Odessa maîtrise plusieurs formes verbales: le présent, le passé composé, l'imparfait, le plus-que-parfait et le futur périphrastique. Pour le présent et l'imparfait, $92 \%$ des occurrences observées pendant les deux mois précédant son séjour en Californie sont formées avec les pronoms je, tu, il/elle et on. Pour ces personnes les terminaisons du présent et celles de l'imparfait sont identiques à l'oral. Pour le passé composé et le plusque-parfait, l'auxiliaire (avoir ou être), au présent ou à l'imparfait, varie selon la personne. Le verbe sous la forme du participe passé, qui est de plus homonyme de l'infinitif pour les verbes en -er, suit l'auxiliaire. Pour le futur périphrastique le verbe aller, marqué en personne, se trouve placé avant le verbe à l'infinitif. Pour tous ces temps, la position préverbale joue un rôle morphologique pour l'indication du temps et de la personne plus important que les terminaisons post-verbales. La saillance attribuée par Odessa à la position pré-verbale en français semble avoir été transférée à l'anglais. Cela explique la précocité de l'auxiliaire dans les productions anglaises et le retard dans les terminaisons post-verbales (-ing, -ed, -en).

\section{Conclusion}

En dépit du fait que cette fillette a été exposée aux deux langues depuis la naissance, la langue française est très dominante dans sa production en français au début du séjour. La relation entre la production en anglais et en français change beaucoup pendant son séjour dans un pays anglophone. Son anglais progresse très rapidement et atteint à la fin du séjour un niveau proche de celui d'un monolingue d'âge équivalent. Cependant, la trajectoire de développement pour atteindre ce niveau diffère considérablement de celle d'un enfant monolingue. Cette étude souligne l'attention à accorder à la production en situation dans l'étude de l'acquisition de deux langues pendant l'enfance. 


\section{BIBLIOGRAPHIE}

BATES, E., I. BRETHERTON, \& L. SNYDER. (1988) From First Words to Grammar: Individual Differences and Dissociable Mechanisms. Cambridge University Press, Cambridge.

BLOOM, L., P. LIGHTBOWN \& L. HOOD. (1974) Imitation in language development: if, when and why? Cognitive Psychology 6: 380-420. Réimprimé 1991. In Bloom, L., éd. Language development from two to three. Cambridge University Press, Cambridge. 399-433.

BLOOM, L., K. LIFTER \& J. HAFITZ. (1980) Semantics of verbs and the development of verb inflection in child language. Language 56: 386-412.

BROWN, R. (1973) A first language, the early stages. Harvard University Press, Cambridge, MA.

CHAPMAN, R. (1981) Mother-child interaction in the second year of life. In R. Schiefelbusch \& D. Bricker, éds., Early language: Acquisition and intervention. University Park Press, Baltimore, 201-250. COMRIE, B. (1976) Aspect. Cambridge University Press, Cambridge.

CREISSELS, D. (1995) Eléments de syntaxe générale. PUF, Paris.

CROFT, W. (1990) Typology and universals. Cambridge University Press, Cambridge.

CROMER, R. (1968) The development of temporal reference during the acquisition of language. Doctoral dissertation, Harvard University.

CUNNINGHAM, C. E. REULER, J. BLACKWELL \& J. DECK. (1981) Behavioral and linguistic developments in the interactions of normal and retarded children with their mothers. Child Development 52: 62-70. DE BOYSSON-BARDIES, B., L. SAGART \& C. DURAND. (1984) Discernible differences in the babbling of infants according to target language. Journal of Child Language 11: 1-15.

DE VILLIERS, J. G. \& P. A. DE VILLIERS. (1973) A cross-sectional study of the acquisition of grammatical morphemes. Journal of Psycholinguistic Research $2: 267-278$.

DE VILLIERS, J. G. \& P. A. DE VILLIERs. (1978) Language Acquisition. Harvard University Press, Cambridge, Mass.

DE VILLIERS, J. G. \& P. A. DE VILLIERs. (1985) The acquisition of English. In D. I. Slobin, éd., The crosslinguistic study of language acquisition. Volume 1: The data. Lawrence Erlbaum Associates, Hillsdale, N.J., 27-139.

DE HOUWER, A. (1990) The acquisition of two languages from birth: a case study. Cambridge University Press, Cambridge

FILLMORE, L. (1976) The second time around: cognitive and social strategies in second language acquisition. Ph.D. Dissertation, Stanford University.

FLETCHER, P. (1985) A child's learning of English. Basil Blackwell, London .

GIVON, T. (1984) Syntax: A functional-typological introduction, vol. 1. John Benjamins, Amsterdam.

GROSJEAN, F. (1982) Life with two languages. Harvard University Press, Cambridge, Mass.

HAKUTA, K. (1974) Prefabricated patterns and the emergence of structure in second language acquisition. Language Learning 24: 287-297. 
HAKUTA, K. (1976) Becoming bilingual: A case study of a Japanese child learning English. Language Learning 26:321-351.

HAKUTA, K. (1986) Mirror of language: The debate on bilingualism. Basic Books, Inc., New York. HOPPER, P. (1979) Aspect and foregrounding in discourse. In T. Givon, éd., Discourse and syntax: Syntax and semantics 12. Academic Press, New York.

HUANG, J. and E. НATCH. (1978) A Chinese child's acquisition of English. In E. Hatch, éd., Second language acquisition: A book of readings. Newbury House, Rowley, Mass.

HYLTENSTAM, K. \& Å. VIBERG. (1993) Linguistic progression and regression: an introduction. In K. Hyltenstam \& Å. Viberg, éds., Progression and regression in language. Cambridge University Press, Cambridge. 3-36.

JISA, H. (1989) The process of language replacement. Actes du Symposium AILA/CILA : Minorisation Linguistique et Interaction, Commission Interuniversitaire Suisse de Linguistique Appliquée.

LEOPOLD, W. F. (1939-1949) Speech development of a bilingual child (4 volumes). Northwestern University Press, Evanston, Illinois.

LYONS, J. 1977. Semantics, vol. II. Cambridge University Press, Cambridge.

MARCHMAN, V. \& L. BATES. (1994) Continuity in lexical and morphological development: A test of the critical mass hypothesis. Journal of Child Language 21: 339-366.

MCLAUGHLin, B. (1984) Second Language Acquisition in Childhood. Volume 1: The Preschool Years. Lawrence Erlbaum, Hillsdale, N.J.

MCLAughuin, B. (1985) Second Language Acquisition in Childhood. Volume 2: School-aged Children. Lawrence Erlbaum, Hillsdale, N.J.

MEHLER, J., G. LAMBERTZ, P. JUSCZYK \& C. AMIEL-TISON. (1986) Discrimination de la langue maternelle par le nouveau-né. Académie des Sciences 3: 637-40.

MEISEL, J. M. (1989) Early differentiation of languages in bilingual children. In K. Hyltenstam and L. Obler, eds., Bilingualism across the lifespan: aspects of acquisition, maturity and loss. Cambridge University Press, Cambridge, MA.

MEISEL, J. M. (1991) Principles of universal grammar and strategies of language learning: some similarities and differences between first and second language acquisition. In L. Eubank, éd., Point counterpoint: Universal grammar in the second language. John Benjamins, Amsterdam.

MOERK, E. L. (1977) Pragmatic and semantic aspects of early language development. University Park Press, Baltimore, Maryland.

PADILLA, A. \& K. LINDHOLM. (1984) Child bilingualism: the same old issues revisited. In J. L. Martinez \& R. H. Mendoza, éds., Chicano psychology. Academic Press, Orlando, 369-408.

PARODI, T. (1990) The acquisition of word order regularities and case morphology. In Meisel, J. M., éd., Two first languages. Foris, Dordrecht, 157-192.

PETERS, A. M. (1983) The units of language acquisition. Academic Press, New York.

RAMER, A. (1976) The function of imitation in child language. Journal of Speech and Hearing Research 19: 700-717.

RETHERFORD, K. S., B. C. SCHWARTZ \& R. S. CHAPMAN. (1981) Semantic roles and residual grammatical categories in mother and child speech: Who tunes into whom? Journal of Child Language 8: 583-608. 
RICHARDS, B. J. (1990) Language development and individual differences: a study of auxiliary verb learning . Cambridge University Press, Cambridge.

RONDAL, J.A. (1983) L'interaction adulte-enfant et la construction du langage. Pierre Mardaga, Bruxelles.

SCHLYTER, S. (1990) The acquisition of tense and aspect. In Meisel, J. M., éd., Two first languages. Foris, Dordrecht, 87-121.

SCHLYTER, S. (1993) The weaker language in bilingual Swedish-French children. In K. Hyltenstam \& A. Viberg, éds., Progression and regression in language: sociocultural, neuropsychological, and linguistic perspectives. Cambridge University Press, Cambridge, MA., 289-308.

SCHLYTER, S. \& G. HÅKANSSON. (1994) Word order in Swedish as the first language, second language and weaker language in bilinguals. Scandinavian working papers on bilingualism 9: 49-66.

SEITZ, S. \& C. STEWART. (1975) Imitations and expansions: some developmental aspects of motherchild communications. Developmental Psychology 11: 763-768.

SELIGER, H. (1989) Deterioration and creativity in childhood bilingualism. In K. Hyltenstam \& L. K. Obler, éds., Bilingualism across the lifespan: aspects of acquisition, maturity and loss. Cambridge University Press, Cambridge, MA, 173-184.

VENDLER, Z. (1967) Verbs and times. In Z. Vendler, Linguistics in philosophy, Cornell University Press: Ithaca, New York, pgs. 97-121.

WELLS, G. (1985) Language development in the pre-school years. Cambridge University Press, Cambridge.

\section{NOTES}

1. Je remercie très sincèrement Suzanne Schlyter pour de nombreuses discussions et suggestions portant sur ce point.

\section{RÉSUMÉS}

Cette étude de cas souligne le rôle de la production dans le développement de la langue faible chez un enfant bilingue français-anglais. En dépit du fait que l'enfant était exposé aux deux langues depuis la naissance, le français est très dominant dans la production. Lors d'un séjour de deux mois en pays anglophone, l'anglais se développe rapidement. Nous retraçons en particulier l'évolution de la forme AUX V-ing qui montre une trajectoire très différente de celle des enfants monolingues anglophones. Nous attribuons le développement atypique à l'influence du français, la langue dominante.

Cette étude de cas souligne le rôle de la production dans le développement de la langue faible chez un enfant bilingue français-anglais. En dépit du fait que l'enfant était exposé aux deux langues depuis la naissance, le français est très dominant dans la production. Lors d'un séjour de deux mois en pays anglophone, l'anglais se développe rapidement. Nous retraçons en particulier 
l'évolution de la forme AUX V-ing qui montre une trajectoire très différente de celle des enfants monolingues anglophones. Nous attribuons le développement atypique à l'influence du français, la langue dominante.

\section{AUTEUR}

HARRIET JISA

Dynamique du Langage (CNRS UMR 9961) \& Université Lumière - Lyon 2, Dynamique du Langage (UMR 9961 CNRS), harriet.jisa@univ-lyon2.fr 